\title{
Improved Ultrastructural Preservation of the Drosophila Neuromuscular Junction by a Combination of Chemical Fixation, High Pressure Freezing and Freeze
} Substitution

\author{
Nirmala A. Iyer ${ }^{1 *}$ and Patricia K. Rivlin ${ }^{1}$ \\ ${ }^{1 .}$ Electron Microscopy Shared Resource, Janelia Research Campus, Ashburn, VA, USA. \\ * Corresponding author: iyern@janelia.hhmi.org
}

The Drosophila larval neuromuscular junction (NMJ) is a model system for studying synaptic structure, function and development. For over 25 years, important insights have been gleaned from electron microscopic (EM) studies of the fly NMJ. While most studies employed conventional EM methods using chemical fixatives, a few recent studies have applied high pressure freezing (HPF) and freeze substitution (FS) techniques [1,2,3].

$\mathrm{HPF} / \mathrm{FS}$ is a powerful technique for preserving ultrastructure at the near-to-native state. In particular, HPF/FS has been used to preserve difficult-to-fix neuronal tissues in dissected brain or intact organisms such as Drosophila larvae $[1,2,3,4,5,6]$. In cases where tissue is prone to dissection damage and ultrastructural degradation, aldehyde fixation prior to HPF/FS has been shown to be a good alternative to HPF/FS alone, preserving ultrastructure better than chemical fixation alone [7].

We developed a novel protocol for HPF/FS of the Drosophila larval NMJ. Unlike previous protocols for the NMJ, we employed a gentle chemical fixation prior to HPF/FS. Briefly, the larval bodywall was dissected and pre-fixed in $0.2 \%$ glutaraldehyde and $4 \%$ paraformaldehye. The bodywall was trimmed with a biopsy punch to fit into HPF planchettes, and frozen in a Wohlwend HPF. Freeze substitution was performed with a cocktail containing $1 \% \mathrm{OsO} 4,0.2 \% \mathrm{UA}, 3 \% \mathrm{H} 2 \mathrm{O}$ in acetone. Following FS, tissue was dehydrated, infiltrated and embedded in Epon. Ultrathin sections were collected and post-stained with UA and lead. Transmission electron microscopic (TEM) images were collected using a Tecnai Spirit T12 at $80 \mathrm{kV}$.

Our TEM results reveal well preserved synapses and other organelles including multivesicular bodies, subsynaptic reticulum (postsynaptic membrane system), and extracellular vesicles (EVs) (Figure 1). Like previous HPF/FS studies of intact larvae, we observed filaments emanating from the presynaptic density (also called T-bar) as well as filaments linking synaptic vesicles. Interestingly, although aldehyde fixation has been proposed to alter synapse morphology, we observed well preserved ultrastructure when combining aldehyde fixation with HPF. Like Sosinksky et al. [7], we suggest that chemical fixation with aldehyde does not necessarily degrade native morphology.

Recently, the fly NMJ has emerged as a model to study the role of EVs in the nervous system [8]. To our knowledge, this is the first example of EVs in the NMJ processed with HPF/FS. In summary, we have developed a protocol employing HPF/FS which is suitable for visualizing the near-to-native ultrastructure of synapses as well as EVs in Drosophila. 
References:

[1] W Fouquet et al., J. Cell Biol. 186 (2009), p. 129.

[2] W Jiao et al., J. Struct. Biol. 172 (2010), p. 389.

[3] Z Hong et al., J. Neurogenetics 30 (2016), p. 237.

[4] P Rostaing et al., Eur. J. Neurosci. 24 (2006).

[5] L Siksou et al., Journal of Neuroscience 27 (2007), p. 6868.

[6] N Korogod, CCH Petersen and GW Knott, Elife 4 (2015), p. 241.

[7] G Sosinsky et al., J. Struct. Biol. 161 (2008), p. 359.

[8] V Budnik, C Ruiz-Canada, F Wendler, Nature Reviews Neurosci. 17 (2015), p. 160.

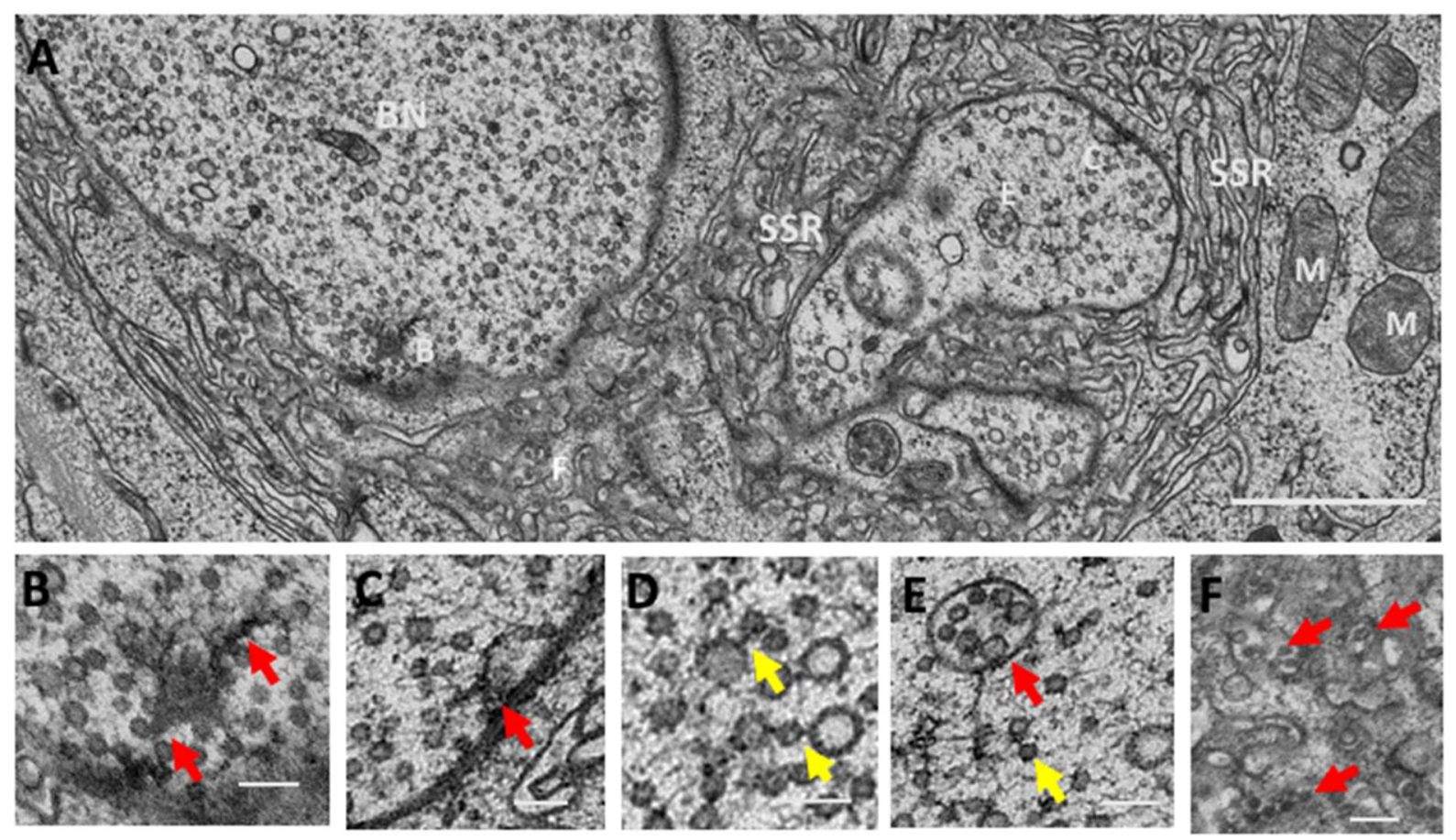

Figure 1. Ultrastructure of Drosophila $3^{\text {rd }}$ instar larval neuromuscular junction preserved by chemical fixation, HPF and FS. (A) TEM image of NMJ. Synaptic terminal or bouton (BN) is surrounded by subsynaptic reticulum (SSR), a postsynaptic membrane system. Mitochondria (M). Organelles marked by B, C, E, and F are shown at higher magnification in corresponding panels below. (B) Filamentous presynaptic density (arrow) as seen in a tangential view. (C) Transverse view of T-shaped presynaptic density (arrow). (D) Well preserved filaments (arrows) link synaptic vesicles. (E) Multivesicular body (red arrow) and synaptic vesicles (yellow arrow), (F) Extracellular vesicles are visible within the subsynaptic reticulum invaginations. Scale bars: $1 \mu \mathrm{m}(\mathrm{A}), 100 \mathrm{~nm}(\mathrm{~B}, \mathrm{C}, \mathrm{D}, \mathrm{E}, \mathrm{F})$. 